\title{
Interval-valued Symbolic Representation based Method for Off-line Signature Verification
}

\author{
Srikanta Pal \\ School of ICT, Griffith \\ University, Australia \\ srikanta.pal@griffithuni.edu.au
}

\author{
Alireza Alaei \\ Laboratoire d'Informatique, \\ Université François-Rabelais, \\ Tours, France \\ alireza20alaei@yahoo.com
}

\author{
Umapada Pal \\ CVPR Unit, Indian Statistical \\ Institute \\ Kolkata, India \\ umapada@isical.ac.in
}

\author{
Michael Blumenstein \\ School of ICT, Griffith \\ University, Australia \\ m.blumenstein@griffith.edu.au
}

\begin{abstract}
The objective of this investigation is to present an interval-symbolic representation based method for offline signature verification. In the feature extraction stage, Connected Components (CC), Enclosed Regions (ER), Basic Features (BF) and Curvelet Feature (CF)-based approaches are used to characterize signatures. Considering the extracted feature vectors, an interval data value is created for each feature extracted from every individual's signatures as an intervalvalued symbolic data. This process results in a signature model for each individual that consists of a set of interval values. A similarity measure is proposed as the classifier in this paper. The interval-valued symbolic representation based method has never been used for signature verification considering Indian script signatures. Therefore, to evaluate the proposed method, a Hindi signature database consisting of 2400 (100x24) genuine signatures and $3000(100 \times 30)$ skilled forgeries is employed for experimentation. Concerning this large Hindi signature dataset, the highest verification accuracy of $91.83 \%$ was obtained on a joint feature set considering all four sets of features, while $\mathbf{2 . 5 \%}$, 13.84\% and 8.17\% of FAR (False Acceptance Rate), FRR (False Rejection Rate), and AER (Average Error Rate) were achieved, respectively.
\end{abstract}

Keywords-Off-line signature verification; Interval-valued symbolic representation; Similarity measure; FAR; FAR; AER.

\section{INTRODUCTION}

Signatures are widely used as proof of identity in our daily lives. They have been accepted as an official means to verify personal identity for legal purposes on many documents such as cheques, credit cards, and wills. Considering the large number of signatures handled daily through visual inspection by people, the construction of a robust and accurate automatic system to process such a huge volume of signatures has many potential benefits for ensuring authenticity of signatures and reducing fraud and other crimes. Automatic signature recognition/verification by computers has received wide research interests in the field of pattern recognition. There are two different ways to recognize a signature: verification and identification. Verification involves confirming or denying a person's claimed signature whereas identification decides the signature group among the number of groups that the claimed signature belongs to.

A signature verification system, and the associated techniques used to solve the inherent problems of authentication, can be divided into two categories: (a) on-line
[1] and (b) off-line [3] methods. Online methods measure temporal and sequential data by utilizing intelligent algorithms [2] whereas off-line methods [3] use an optical scanner to obtain handwriting data written on paper and they appear in a static format [4].

There are many research works in the literature for offline signature verification [3-9]. Nguyen and Blumenstein [5] presented a global feature-based approach, where the writer energy to create the signature, and horizontal and vertical projection profiles of the signature have been used as features. In another signature verification technique [6], the authors proposed a hybrid approach, where local features with Gaussian mixture models and global features with kNN were used. Their experiment was conducted on ICDAR2009 and ICFHR2010 (4NSigComp) datasets. In their concluding remarks it was mentioned that local features are predominantly better. Ferrer et al. [7] proposed a new method for the generation of synthetic offline signatures. The experimental protocol of their study includes the comparison of both types of signatures in terms of: i) performance evaluation of two competitive and totally different verification systems; and ii) visual appearance according to human observers. The experimental results showed that a high similarity exists between synthetically produced and human generated samples. Solar et al. [8] extracted local interest points and computed descriptors for off-line signature verification. Local interest points were detected in the signature image and local descriptors were computed in the neighbourhood of these points. The local descriptors were subsequently compared using local and global matching procedures. The verification is carried out using a Bayesian classifier. In another study, Malik et al. [9] presented the results of the ICDAR2013 competition on signature verification and writer identification for online/off-line skilled forgeries (SigWiComp2013) jointly organized by pattern recognition researchers and forensic handwriting examiners. Four tasks were defined where the systems had to perform Dutch offline signature verification, Japanese offline signature verification, Japanese online signature verification, and Dutch writer identification.

From the literature surveyed, it is noted that there is some impressive progress in the field of signature verification. The methods presented in SigWiComp2013 indicate that the problem of signature identification/verification still remains a challenging problem [9], since accurate authentication is a difficult task. Moreover, very few research works, which 
employ signatures of Indian scripts, have been deliberated in the field of non-English based signature identification/ verification [11-14]. To fill this gap, a verification method for off-line Hindi signatures based on symbolic representation model considering some state-of-the-art features is proposed. This investigation leads us to incorporate a new model-based technique for signature verification.

The concept of symbolic data analysis [16] has been used in many applications of document image analysis such as document classification, character recognition and signature verification [17-19]. To demonstrate the applicability of symbolic data analysis, a new interval-based signature verification method is presented in this research work. In this paper, different signature samples captured from each individual are represented using an interval-valued symbolic data vector. This process provides only one representative model for each individual's handwritten signature instead of many feature vectors, which represent different signatures of an individual. The applicability of the proposed method is demonstrated using Hindi signatures for experimentation, and promising results are obtained.

The remainder of this paper is structured as follows. Section II explains the proposed method. The experimental settings and database details are presented in Section III. The experimental results are presented in Section IV. In Section V, the performance of comparison is discussed. Finally, conclusions are presented in Section VI.

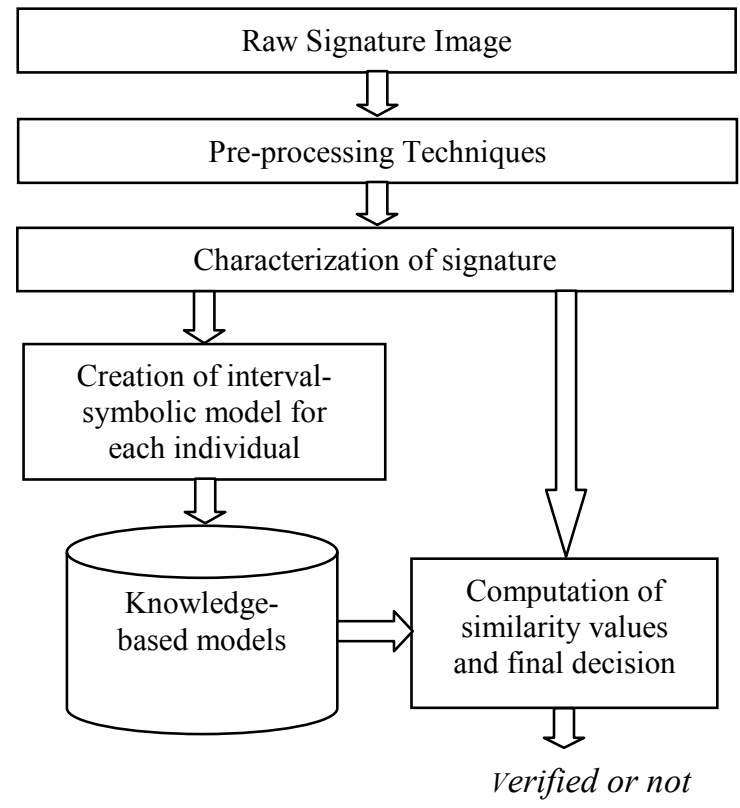

Fig. 1. Overview of the proposed method.

\section{PRoposed METHOD}

An overview of our proposed signature verification method is shown in Fig. 1. The proposed method includes the following steps: a) pre-processing, b) characterization of signatures, c) creation of an interval-valued symbolic model for each individual, and d) computation of similarity values and final decision. Each step is detailed in the following subsections.

\section{A. Pre-processing}

The signatures to be processed by the system are required in a digital image format. At the very beginning, the images were captured in 256 level grey-scale at 300 dpi and stored in TIFF format (Tagged Image File Format). In the preprocessing step, a histogram-based threshold technique was applied for binarization to convert digitized grey-level image to a two-tone image. A mean filter is also employed on the signature images to remove noise from the signature images.

\section{B. Characterization of signature}

Feature extraction is an important step in any pattern recognition system. Since, our objective in this research work is proposing a new model for signature verification rather than proposing some new features for characterizing signatures, a set of simple features mainly introduced in the literature for writer verification $[15,20,21,22]$ is considered. The features used in this research are of four types: i) Connected Components (CC), ii) Enclosed Regions (ER), iii) Basic Features (BF), and iv) Curvelet Feature (CF) based features. In total, 24 features are extracted from each signature image to characterize the signature. Details of each feature extraction techniques are described below.

Connected Components (CC): Individuals' signatures generally consist of two or more words (first name, middle name, last name etc.). To provide signatures in Hindi, people write their full name and this full name is treated as the signature. Therefore, the Hindi signature consists of two or more words. A whole word is sometime written in a single, continuous stroke by some people, whereas the word is also broken up into a number of components by other people. The connected component feature is subjected to model this writing style. Each connected component $C$ is described by its bounding box $\left\{\left(x_{1}\left(C_{i}\right), y_{1}\left(C_{i}\right)\right),\left(x_{2}\left(C_{i}\right), y_{2}\left(C_{i}\right)\right)\right\}$, where $\left(x_{1}\left(C_{i}\right), y_{1}\left(C_{i}\right)\right)$ and $\left(x_{2}\left(C_{i}\right), y_{2}\left(C_{i}\right)\right)$ are the coordinates of the left-lower and right-upper corner of the bounding box of $C_{i}$, respectively. The average distance between two successive bounding boxes is computed from all connected components of a signature. In this regard, all connected components according to their $x_{1}$ value are ordered. Given the ordered list $\left(C_{1}, C_{2}, \cdots, C_{n}\right)$, the average value of $\left(x_{1}\left(C_{i+1}\right)-\right.$ $\left.x_{2}\left(C_{i}\right)\right)$ is calculated. This quantity is used as a feature that is potentially useful for signature verification [20].

The average distance of two consecutive words and the average within-word distance of connected components are considered for next two features. To compute these two features, a clustering technique is applied that groups connected components together if they are likely to belong to the same word. This clustering procedure uses a threshold $t$ on the distance of two consecutive connected components, $C_{i}$ and $C_{i+1}$. If $\left(x_{1}\left(C_{i+1}\right)-x_{2}\left(C_{i}\right)\right) \leq t$ then it is assumed that $C_{i}$ and $C_{i+1}$ belong to the same word. Otherwise, $C_{i}$ is considered to be the last component of a word $w_{j}$ and $C_{i+1}$ is considered to be the first component of the following word $w_{j+1}$. Other features derived from the connected components are the average, median, and standard deviation of the length $\left(x_{2}\left(C_{i}\right)-\right.$ 
$\left.x_{1}\left(C_{i}\right)\right)$ of connected components $C_{i}$ in a signature, and the average number of black-to-white transitions within each connected component [20].

Enclosed Regions (ER): If the closed loops occurring in handwritten signature are analyzed, certain properties that are specific to individual writers are observed. For example, the shape of loops of some writers is circular whereas the shape of loops is more elliptical for other writers. To simplify the computational procedures, the loops are not analyzed directly, the blobs that are enclosed by the loops are considered for analyzing. These blobs can be easily computed by standard region growing algorithms. The first feature extracted from the blobs is the average of the form factor taken over all blobs of a signature. The second feature is the average roundness of blobs in a signature image. The last feature is the average size of the blobs in a signature. Details of these features can be found in [20]. For a graphical illustration, Fig. 2 and Fig. 3 are provided. In Fig. 2, a signature sample from the database used in the experiments is shown. In Fig. 3, the blobs enclosed by the loops corresponding to Fig. 2 are displayed.

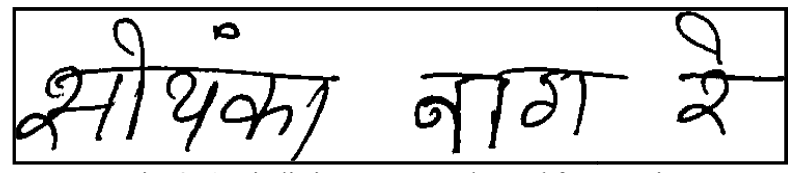

Fig. 2. A Hindi signature sample used for experiment

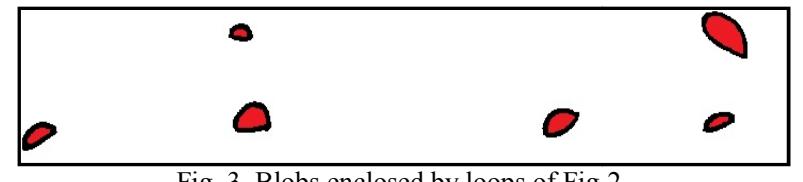

Fig. 3. Blobs enclosed by loops of Fig 2.

Basic Features $(B F)$ : In addition to the features described in the previous sections, some features that were used already in [15-21] are included in the experiments. These features correspond to writing skew and slant, the height of three main writing zones, and the width of the writing. Computational procedures for extracting these features can be found in [15].

Curvelet Feature $(\mathrm{CF})$ : Curvelets are an appropriate basis for representing images (or other functions), which are smooth apart from singularities along smooth curves, where the curves have bounded curvature, i.e. where objects in the image have a minimum length scale [10]. Curvelets take advantage of this property, by defining the higher resolution Curvelets to be more elongated than the lower resolution Curvelets. The representation that Curvelets can produce are significantly sparser compared with other wavelet transform. This property can be defined by considering the best approximation of a geometrical test image that can be represented using only $n$ wavelets, and analysing the approximation error as a function of $n$. For a Fourier transform, the squared error decreases only as $O(1 / \sqrt{n})$ for a wide variety of wavelet transforms, including both directional and non-directional variants, the squared error decreases as $O(1 / n)$. The extra assumption underlying the Curvelet transform allows it to achieve $O\left((\log (n))^{3} / n^{2}\right)$

\section{Creation of Interval-valued symbolic model for each individual}

In the literature, objects/patterns are generally characterized using a set of single value variables called as feature set. The feature vectors extracted for different objects constitute a dataarray, where each cell $(i, j)$ comprises the value of feature $j$ for object $i$. Apart from its simple representation, this kind of modeling cannot take into account the variability and/or uncertainty of feature values. To represent the variability and distribution of feature values in a specific class object, intervaland histogram-valued symbolic variables have been presented in the domain of symbolic data analysis [16]. An object or a set of similar objects is described by interval-valued variables. An interval-variable can be defined using minimum and maximum values of a set of values as follows.

Formally, let $X$ be a continuous variable defined on a finite support $[\underline{x}, \bar{x}]$ where $\underline{x}$ and $\bar{x}$ are the minimum and maximum values of $X$ respectively.

In this research work, using the above-mentioned concept, an interval-valued symbolic data is initially created to model each feature of every individual's signature extracted from the training data. The mean and standard deviation of each feature are also considered. Consequently, an individual's signature is represented by a number of interval-valued data, the mean and the standard deviation computed for all the features used to characterize signatures. To formulate this concept, specific mathematical descriptions are further provided in the following.

Let $S_{j}=\left\{s_{j}^{1}, s_{j}^{2}, \ldots, s_{j}^{m}\right\}$ be a set of $m$ samples from a signature class $C_{j} . F_{j}^{i}=\left\{f_{j 1}^{i}, f_{j 2}^{i}, \ldots, f_{j n}^{i}\right\}$ illustrates a feature vector of size $n$ extracted from the $i^{\text {th }}$ sample of $S_{j}$ say $s_{j}^{i}$. For the $k^{\text {th }}$ feature $f_{j k}^{\circ}$ in every class $C_{j}$, we compute the statistical mean $m_{j k}$ and standard deviation $s t d_{j k}$. Considering $m_{j k}$ and $s t d_{j k}$ (the mean and standard deviation) of the $k^{\text {th }}$ feature of class $j$, the lower bound and upper bound values of $f_{j k}^{\circ}$ are computed in the following to be used for creation of an interval-value for feature $k$ of class $j$.

$$
\begin{aligned}
& s f_{j k}=\left(\left[f_{j k}^{-}, f_{j k}^{+}\right], m_{j k}, s t d_{j k}\right) \\
& f_{j k}^{-}=m_{j k}-\lambda_{j} \times s t d_{j k} \\
& f_{j k}^{+}=m_{j k}+\lambda_{j} \times s t d_{j k} \\
& m_{j k}=\operatorname{Mean}\left(f_{j k}^{\circ}\right) \\
& \operatorname{std}_{j k}=\operatorname{StandardDeviation}\left(f_{j k}^{\circ}\right)
\end{aligned}
$$

where $s f_{j k}$ is a symbolic representation of the $k^{\text {th }}$ feature of class $j\left(C_{j}\right)$ composed of an interval-value and 2 continues values (mean and standard deviation). $\lambda_{j}$ is a parameter which should be tuned during the training phase. As a result, symbolic representation of $C_{j}$ called $S y m C_{j}$ is composed of $n$ symbolic features $(s f)$ and defined as follows:

$$
\operatorname{symC}_{j}=\left\{s f_{j 1}, s f_{j 2}, \ldots, s f_{j n}\right\}
$$

Considering $q$ classes in a particular problem, the complete interval symbolic representation of the problem is shown in Table I. 
TABLE I. INTERVAL-SYMBOLIC REPRESENTATION OF A $q$-CLASS PROBLEM WITH $n$ FEATURES BASED ON THE PROPOSED APPROACH.

\begin{tabular}{|c|c|c|c|}
\hline Class & $\boldsymbol{s f}_{1}$ & $\cdots$ & $\boldsymbol{s}_{n}$ \\
\hline $\operatorname{Sym}_{\boldsymbol{n}} C_{1}$ & $\left(\left[f_{11}^{-}, f_{11}^{+}\right], m_{11}, s t d_{11}\right)$ & $\ldots$ & $\left(\left[f_{1 n}^{-}, f_{1 n}^{+}\right], m_{1 n}, s t d_{1 n}\right)$ \\
\hline $\operatorname{Sym}_{2} C_{2}$ & $\left(\left[f_{21}^{-}, f_{21}^{+}\right], m_{21}, s t d_{21}\right)$ & $\cdots$ & $\left(\left[f_{2 n}^{-}, f_{2 n}^{+}\right], m_{2 n}, s t d_{2 n}\right)$ \\
\hline$\ldots$ & $\ldots$ & $\cdots$ & $\ldots$ \\
\hline $\operatorname{Sym}_{j}$ & $\left(\left[f_{j 1}^{-}, f_{j 1}^{+}\right], m_{j 1}, s t d_{j 1}\right)$ & $\ldots$ & $\left(\left[f_{j n}^{-}, f_{j n}^{+}\right], m_{j n}, s t d_{j n}\right)$ \\
\hline$\ldots$ & $\ldots$ & $\ldots$ & $\ldots$ \\
\hline $\operatorname{Sym}_{C_{q}}$ & $\left(\left[f_{q 1}^{-}, f_{q 1}^{+}\right], m_{q 1}, s t d_{q 1}\right)$ & $\cdots$ & $\left(\left[f_{q n}^{-}, f_{q n}^{+}\right], m_{q n}, s t d_{q n}\right)$ \\
\hline
\end{tabular}

D. Computation of similarity values and verification process

Euclidian, City-block and Mahalanobis distances are some frequent and simple distance measures used in the literature for computing similarity/dissimilarity between two feature vectors. Since, in this research work the proposed representation model for each signature class is based on the interval-values and each feature extracted from a test signature is a single numerical value, a specific distance measure is proposed to compute the similarity between every intervalsymbolic model and a test sample. The similarity $\operatorname{Sim}\left(F_{T}\right.$, Sym $C_{j}$ ) between a test sample $(T)$ and a symbolic reference of a particular class $j\left(\mathrm{Sym}_{j}\right)$ is computed as follows:

$$
\rho_{j i}=\left\{\begin{array}{cc}
0 & \operatorname{Sim}\left(F_{T}, \operatorname{Sym}_{j}\right)=\left(\sum_{i=1}^{n} P_{j i}\right) / n \\
1 & \text { if } F_{T i}<f_{j i}^{-} \text {or } F_{T i}>\mathrm{f}_{\mathrm{ji}}^{+} \\
& \text {if } M_{j k}-\lambda_{j} \times S T D_{j k} \leq F_{T i} \text { and } \\
0.5 & F_{T i} \leq M_{j i}+\lambda_{j} \times S T D_{j i} \\
& \text { if } f_{\mathrm{ji}}^{-} \leq F_{T i}<M_{j i}-\lambda_{j} \times S T D_{j i} \text { or } \\
& M_{j i}+\lambda_{j} \times S T D_{j i}<F_{T i} \leq f_{j i}^{+}
\end{array}\right.
$$

Based on the similarity value $\left(\operatorname{Sim}\left(F_{T}, \operatorname{Sym}_{j}\right)\right)$ an acceptance threshold $(j)$ is defined as the confidence value for class $C_{j}$. The value of ${ }_{j}$ is tuned during the training step in order to obtain the most reliable results. Based on the verification results on the training data, ${ }_{j}$ is defined as follows:

$\theta_{j}=\operatorname{Mean}\left(\operatorname{Sim}\left(F_{T}^{i}, \operatorname{Sym} C_{j}\right)+\operatorname{STD}\left(\operatorname{Sim}\left(F_{T}^{i}, \operatorname{Sym} C_{j}\right)\right)\right.$

where $i$ varies from 1 to number of samples in the training set of class $C_{j}$.

\section{DATABASE Details AND EXPERIMENTAL SetTings}

In the field of signature verification, there is a sparseness of publicly available signature databases. The quality of the available databases also varies, as there has been no standard collection protocol. Besides, it is very costly to create a large corpus with different types of forgeries, especially skilled forgeries. As there has been no public signature corpus available for Hindi script, it was necessary to create a database of Hindi signatures. This Hindi signature database consists of 100 sets. Each set consists of 24 genuine signatures and 30 skilled forgeries. A total number of 2400 genuine and 3000 skilled forgery signatures were collected from 100 individuals. Some genuine signature samples with their corresponding forgeries are displayed in Table II.
In this experimental method of verification, 16 genuine signatures from each individual were considered for training. No forged signature samples were employed for training. The remaining 8 genuine signatures and 30 skilled forgeries from each individual in the signature dataset were considered for testing purposes.

TABLE II. GENUINE AND FORGED SIGNATURE SAMPLES OF THREE DIFFERENT CLASSES WRITTEN IN HINDI.

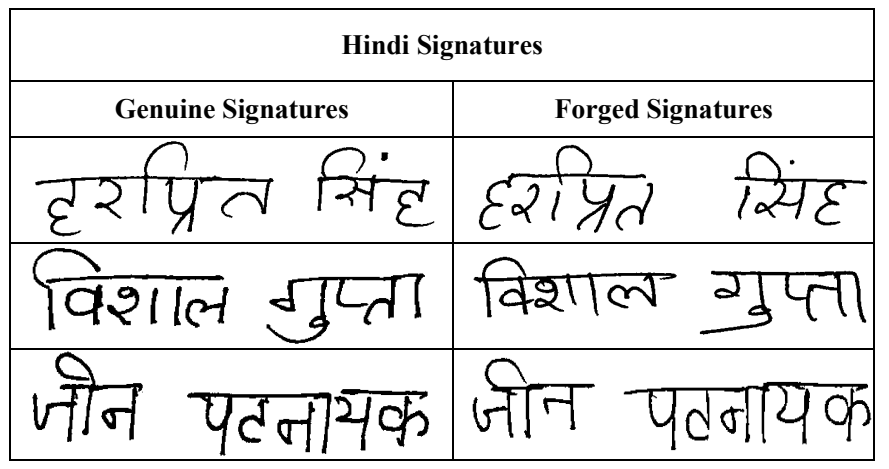

\section{RESUlTS AND DISCUSSION}

The experiment was conducted considering each feature set alone and also different combinations of feature sets. This is done to observe how each feature type performs separately and further how combinations of different types of features improve the verification accuracies. The outcome of the system was measured in terms of FAR (False Acceptance Rate), FRR (False Rejection Rate) and AER (Average Error Rate). The accuracies obtained in these experiments are described and shown in tabular form in the following subsections.

\section{A. Experiment 1:}

In this step of experimentation, CC-based feature set was used to characterize the signatures and build the symbolic interval for each individual. The accuracy was computed for different values of $\lambda_{j}$. The results obtained employing the CCbased features in the experiment with different values of $\lambda_{j}$ for symbolic creation are shown in Table III. Subsequently, the results obtained based on each ER, BF and CF-based features are tabulated in Table IV, V, and VI respectively. From Table III, it is observed that the highest accuracies obtained in the experiment were when $\lambda_{j}$ was considered to be 0 whereas in Table IV, V, and VI, the best results obtained when the values of $\lambda_{j}$ were 1.7, 2.3 and 2.1. The reason is that the values of features extracted based on CC had broadly distributed and lowers and uppers bounds were far from the mean values. However, in the other three feature sets there are not much variation in the feature values in the training sets. To provide wider distributions for the features in those three types of features (ER, BF, and $\mathrm{CF}$ ), mean plus/minus 2 times of standard deviations were used for upper/lower bounds of features.

From this experiment it is further noted that the best performance was obtained from the CF-based features. Using this 10 texture features, around $90 \%$ of signatures were accurately verified. The results obtained based on the CC features are in the second rank with an AER of 10.95. This indicates that more than $89 \%$ of signatures were correctly verified using only $9 \mathrm{CC}$-based features. $\mathrm{BF}$ and ER-based 
features also provided reasonably good results, as the number of BF and ER-based features is only 3 and 2 respectively. BFbased features provided the best AER result of $14.28 \%$.

TABLE III. EXPERIMENTAL ACCURACIES (\%) CONSIDERING CC-BASED FEATURES WITH DIFFERENT VALUES OF LAMDA $\left(\lambda_{J}\right)$.

\begin{tabular}{|l|c|c|c|c|c|c|c|c|}
\hline \multicolumn{1}{|c|}{ Lambda } & $\mathbf{0}$ & $\mathbf{0 . 3}$ & $\mathbf{0 . 6}$ & $\mathbf{0 . 9}$ & $\mathbf{1 . 2}$ & $\mathbf{1 . 7}$ & $\mathbf{2 . 1}$ & $\mathbf{2 . 3}$ \\
\hline FAR & $\mathbf{1 . 3 9}$ & 25.05 & 27.50 & 27.32 & 29.34 & 33.39 & 35.89 & 35.53 \\
\hline FRR & $\mathbf{2 0 . 5 0}$ & 11.92 & 11.74 & 11.26 & 10.61 & 9.42 & 8.50 & 8.50 \\
\hline AER & $\mathbf{1 0 . 9 5}$ & 18.49 & 19.62 & 19.29 & 19.97 & 21.41 & 22.20 & 22.01 \\
\hline
\end{tabular}

TABLE IV. EXPERIMENTAL ACCURACIES (\%) CONSIDERING ER-BASED FEATURES WITH DIFFERENT VALUES OF $\lambda_{s}$

\begin{tabular}{|l|c|c|c|c|c|c|c|c|}
\hline $\begin{array}{l}\text { Lambda } \\
\text { Results }\end{array}$ & $\mathbf{0}$ & $\mathbf{0 . 3}$ & $\mathbf{0 . 6}$ & $\mathbf{0 . 9}$ & $\mathbf{1 . 2}$ & $\mathbf{1 . 7}$ & $\mathbf{2 . 1}$ & $\mathbf{2 . 3}$ \\
\hline FAR & 53.82 & 45.92 & 39.03 & 31.47 & 26.89 & $\mathbf{2 5 . 0 0}$ & 25.89 & 26.58 \\
\hline FRR & 3.53 & 5.03 & 6.32 & 8.03 & 8.66 & $\mathbf{8 . 9 5}$ & 9.26 & 9.74 \\
\hline AER & 28.67 & 25.47 & 22.67 & 19.75 & 17.78 & $\mathbf{1 6 . 9 7}$ & 17.58 & 18.16 \\
\hline
\end{tabular}

TABLE V. EXPERIMENTAL ACCURACIES (\%) CONSIDERING BF-BASED FEATURES WITH DIFFERENT VALUES OF $\lambda_{j}$

\begin{tabular}{|l|c|c|c|c|c|c|c|c|}
\hline Results & $\mathbf{0}$ & $\mathbf{0 . 3}$ & $\mathbf{0 . 6}$ & $\mathbf{0 . 9}$ & $\mathbf{1 . 2}$ & $\mathbf{1 . 7}$ & $\mathbf{2 . 1}$ & $\mathbf{2 . 3}$ \\
\hline FAR & 67.13 & 66.18 & 48.89 & 45.34 & 31.32 & 30.00 & 22.53 & $\mathbf{1 3 . 9 5}$ \\
\hline FRR & 1.76 & 1.95 & 4.61 & 5.26 & 7.76 & 8.00 & 11.26 & $\mathbf{1 4 . 6 1}$ \\
\hline AER & 34.45 & 34.07 & 26.75 & 25.30 & 19.54 & 19.00 & 16.89 & $\mathbf{1 4 . 2 8}$ \\
\hline
\end{tabular}

TABLE VI. EXPERIMENTAL ACCURACIES (\%) CONSIDERING CF-BASED FEATURES WITH DIFFERENT VALUES OF $\lambda_{J}$

\begin{tabular}{|l|c|c|c|c|c|c|c|c|}
\hline $\begin{array}{l}\text { Lambda } \\
\text { Results }\end{array}$ & $\mathbf{0}$ & $\mathbf{0 . 3}$ & $\mathbf{0 . 6}$ & $\mathbf{0 . 9}$ & $\mathbf{1 . 2}$ & $\mathbf{1 . 7}$ & $\mathbf{2 . 1}$ & $\mathbf{2 . 3}$ \\
\hline FAR & 8.89 & 12.66 & 11.34 & 9.16 & 8.18 & 7.92 & $\mathbf{7 . 3 2}$ & 8.08 \\
\hline FRR & 12.32 & 11.26 & 11.53 & 11.95 & 12.45 & 12.79 & $\mathbf{1 2 . 8 4}$ & 12.61 \\
\hline AER & 10.61 & 11.96 & 11.43 & 10.55 & 10.32 & 10.36 & $\mathbf{1 0 . 0 8}$ & 10.34 \\
\hline
\end{tabular}

\section{B. Experiment 2:}

Since in the first experiment, the CF-based provided the best results for the signature verification, in this experimental step, we included each set of feature to the CF-based feature set based on their performance one by one to demonstrate the improvement of the verification results. First, CC-based features were included into CF-based feature set to make a feature set of $19(10+9)$ features for signature characterization and verification purposes. The accuracies, computed for different values of $\lambda_{j}$ are shown in Table VII. From the table it is observed that the highest accuracy obtained in the experiments when $\lambda$ was considered as 1.7. By combining these two feature sets, the verification accuracies were improved and the best AER result of $9.51 \%$ was achieved.

TABLE VII EXPERIMENTAL ACCURACIES OBTAINED COMBINING CF AND CCBASED FEATURES WITH DIFFERENT VALUES OF $\lambda_{J}$.

\begin{tabular}{|l|c|c|c|c|c|c|c|c|}
\hline $\begin{array}{l}\text { Lambda } \\
\text { Results }\end{array}$ & $\mathbf{0}$ & $\mathbf{0 . 3}$ & $\mathbf{0 . 6}$ & $\mathbf{0 . 9}$ & $\mathbf{1 . 2}$ & $\mathbf{1 . 7}$ & $\mathbf{2 . 1}$ & $\mathbf{2 . 3}$ \\
\hline FAR & 0.00 & 5.42 & 6.00 & 5.82 & 5.21 & $\mathbf{5 . 6 1}$ & 7.18 & 8.29 \\
\hline FRR & 20.97 & 14.47 & 14.16 & 13.82 & 14.00 & $\mathbf{1 3 . 4 2}$ & 12.61 & 12.42 \\
\hline AER & 10.49 & 9.95 & 10.08 & 9.82 & 9.61 & $\mathbf{9 . 5 1}$ & 9.89 & 10.36 \\
\hline
\end{tabular}

For the second combination, the feature sets extracted from $\mathrm{CF}, \mathrm{CC}$ and $\mathrm{BF}-$ based approaches were considered to construct a set of $22(10+9+3)$ features for characterizing signatures and creation of symbolic models. The accuracies, obtained in the experimentation are shown in Table VIII. The highest accuracy obtained in the experiments resulted when 1.7 was considered for $\lambda_{j}$ that provided reduction of AER by $1.2 \%$ compared to the CF-based features.

TABLE VIII. EXPERIMENTAL ACCURACIES OBTAINED COMBINING CF, CC AND BF-BASED FEATURES WITH DIFFERENT VALUES OF $\lambda_{l}$.

\begin{tabular}{|l|c|c|c|c|c|c|c|c|}
\hline \begin{tabular}{|l|c|} 
Lambda \\
Results
\end{tabular} & $\mathbf{0}$ & $\mathbf{0 . 3}$ & $\mathbf{0 . 6}$ & $\mathbf{0 . 9}$ & $\mathbf{1 . 2}$ & $\mathbf{1 . 7}$ & $\mathbf{2 . 1}$ & $\mathbf{2 . 3}$ \\
\hline FAR & 0.00 & 3.66 & 4.45 & 4.13 & 3.47 & $\mathbf{3 . 7 6}$ & 4.84 & 5.18 \\
\hline FRR & 20.97 & 14.97 & 14.50 & 14.24 & 14.37 & $\mathbf{1 4 . 0 0}$ & 13.03 & 13.13 \\
\hline AER & 10.49 & 9.32 & 9.47 & 9.18 & 8.92 & $\mathbf{8 . 8 8}$ & 8.93 & 9.16 \\
\hline
\end{tabular}

Finally, an experiment was conducted concerning the combined feature set of all 24 features extracted based on the $\mathrm{CF}, \mathrm{CC}, \mathrm{BF}$ and $\mathrm{ER}$. The accuracies for different threshold values are shown in Table IX.The highest accuracy of $91.83 \%$ $(100-8.17)$ was obtained in this step of experimentation when the $\lambda_{j}$ was set to 1.7 .

From the results obtained using different combinations of feature set shown in Table VII, VIII, and IX, it is also noted that by including each feature set an improvement between $0.57 \%$ and $0.67 \%$ was achieved. This is quite interesting as including a few simple features could improve the performance of the proposed method. Furthermore, these results indicate that these features are suitable to well characterize Hindi signatures.

TABLE IX. EXPERIMENTAL ACCURACIES OBTAINED CONSIDERING CF, CC, FF AND BF-BASED FEATURES WITH DIFFERENT VALUES FOR $\lambda_{J}$.

\begin{tabular}{|l|c|c|c|c|c|c|c|c|}
\hline \begin{tabular}{|l|c|c|c|c|c|c|c|} 
Lambda \\
Results
\end{tabular} & $\mathbf{0}$ & $\mathbf{0 . 3}$ & $\mathbf{0 . 6}$ & $\mathbf{0 . 9}$ & $\mathbf{1 . 2}$ & $\mathbf{1 . 7}$ & $\mathbf{2 . 1}$ & $\mathbf{2 . 3}$ \\
\hline FAR & 0.00 & 2.63 & 3.50 & 3.37 & 2.39 & $\mathbf{2 . 5 0}$ & 3.82 & 3.37 \\
\hline FRR & 20.97 & 15.66 & 14.58 & 14.79 & 14.47 & $\mathbf{1 3 . 8 4}$ & 13.66 & 13.42 \\
\hline AER & 10.49 & 9.14 & 9.04 & 9.08 & 8.43 & $\mathbf{8 . 1 7}$ & 8.74 & 8.39 \\
\hline
\end{tabular}

\section{COMPARISON OF PERFORMANCE}

To compare the performance of the proposed method with other approaches, a Neural Network based approach was considered in this research work. A probabilistic neural network (PNN) with three layers (input, hidden and output layers) was used for the experimentation. The same datasets and feature sets used for training and testing in the proposed interval-symbolic representation method were employed for training and testing of the PNN. The only parameter in the PNN that should be tuned was spread of radial basis function. A number of values ( 30 values) were considered to find the best performance for the PNN. The results obtained based on the PNN are shown in Table X. From Table X, it is clear that the best results $(91.61 \%)$ obtained when the spread of radial was set to 0.01 . The comparison of performances of the proposed method and PNN-based approach is demonstrated in Table XI. Comparing the proposed method and the PNN based approach; a better accuracy $(91.83 \%)$ was obtained from the proposed interval-symbolic representation-based method. Moreover, the proposed interval-valued symbolic based method provided quite better results in terms of FAR metric compared to the PNN-based approach. Smaller amount of false acceptance rate is very important in any authentication system, since it prevents accepting fraud signatures in the system. 
TABLE X. EXPERIMENTAL ACCURACIES OBTAINED FROM THE PNN CONSIDERING ALL FEATURES WITH DIFFERENT VALUES OF SPREAD RADIAL.

\begin{tabular}{|l|c|c|c|c|c|c|c|c|}
\hline $\begin{array}{r}\text { Spread of } \\
\text { Radial }\end{array}$ & $\mathbf{0 . 0 8}$ & $\mathbf{0 . 0 4}$ & $\mathbf{0 . 0 2}$ & $\mathbf{0 . 0 1}$ & $\mathbf{0 . 0 0 8}$ & $\mathbf{0 . 0 0 3}$ & $\mathbf{0 . 0 0 1}$ & $\mathbf{0 . 0 0 0 9}$ \\
\hline Fesults & & & & & & & & \\
\hline FRR & 18.74 & 13.50 & 13.61 & $\mathbf{1 5 . 4 7}$ & 17.71 & 19.29 & 20.50 & 20.58 \\
\hline AER & 3.42 & 5.00 & 4.97 & $\mathbf{1 . 3 2}$ & 0.37 & 0.21 & 0.21 & 0.21 \\
\hline
\end{tabular}

TABLE XI. COMPARISON OF THE RESULTS OBTAINED FROM THE PROPOSED METHOD AND THE PNN BASED APPROACH.

\begin{tabular}{|l|c|c|}
\hline Mesults & $\begin{array}{c}\text { MNN-based } \\
\text { method }\end{array}$ & $\begin{array}{c}\text { Proposed } \\
\text { method }\end{array}$ \\
\hline FAR & 15.47 & $\mathbf{2 . 5 0}$ \\
\hline FRR & 1.32 & $\mathbf{1 3 . 8 4}$ \\
\hline AER & 8.39 & $\mathbf{8 . 1 7}$ \\
\hline
\end{tabular}

A pictorial representation of the AER results obtained based on the proposed interval-valued symbolic representation method and the PNN approach is shown in Fig.4. From Fig.4, it is evident that the performance of proposed interval valuedsymbolic representation method is better than the PNN approach irrespective to different parameters $(\lambda$ and Spread of radial (SR)), as the AER values obtained from the proposed interval-valued symbolic method are smaller than the AER values obtained based on the PNN approach.

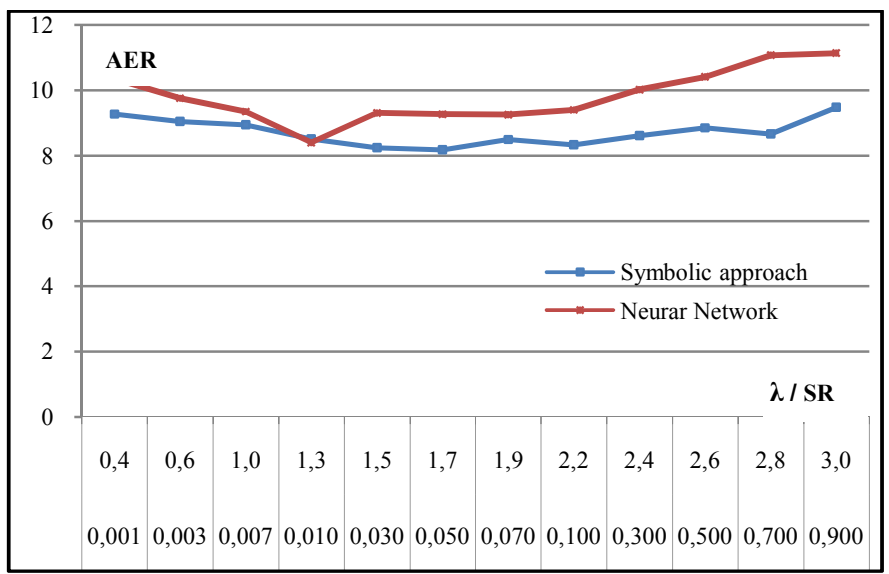

Fig. 4. Performances of the proposed interval-valued symbolic representation approach and the PNN-based method. The first row indicates values of $\lambda$ and the second row presents different values of SR.

\section{CONCLUSION}

This study demonstrates an investigation of the excellent performance of a signature verification approach involving Hindi off-line signatures. An interval-valued symbolic representation-based approach for offline signature verification provides a substantial contribution in the field of signature verification. $\mathrm{CC}, \mathrm{ER}, \mathrm{BF}$ and $\mathrm{CF}$-based methods were used for characterizing signatures. The proposed off-line Hindi signature verification method is a new investigation in the field of Indian-based off-line signature verification. In future, we plan to investigate the application of the proposed method on other non-Indian-script signature verification.

\section{REFERENCES}

[1] S. Emerich, E. Lupu, C. Rusu. "On-line Signature Recognition Approach Based on Wavelets and Support Vector Machines," In Proc. of the
International Conference on Automation Quality and Testing Robotics, pp.1-4, 2010.

[2] A. Kholmatov, B. Yanikoglu, "Identity Authentication using improved online signature verification method," Pattern Recognition Letters, Vol26, pp. 2400-2408, 2005.

[3] M. Kalera, S. Srihari, A. Xu, "Offline signature verification and identification using distance statistics," International Journal of Pattern Recognition and Artificial Intelligence, pp.1339-1360, 2004.

[4] I. Pottier, G. Burel, "Identification and Authentication of Handwritten Signatures with a Connectionist Approach," In Proc. of the IEEE Conference on Neural Networks, pp. 2948-2951, 1994.

[5] V. Nguyen, M. Blumenstein, G. Leedham, "Global Features for the OffLine Signature Verification Problem," In Proc. of the ICDAR, pp. 13001304. 2009.

[6] M. I. Malik, M. Liwicki, A. Dengel, "Evaluation of local and global features for offline signature verification," In Proc. of the International Workshop on Automated Forensic Handwriting Analysis, pp. 26-30, 2011.

[7] M. A. Ferrer, M. Diaz-Cabrera, A. Morales, J. Galbally, M. GomezBarrero, "Realistic Synthetic Off-Line Signature Generation Based on Synthetic On-Line Data," In Proc. of the International Carnahan Conference on Security Technology, pp.1-6. 2013.

[8] J. Ruiz-Del-Solar, C. Devia, P. Loncomilla, F. Concha, "Offline signature verification using local interest points and descriptors," In Proc. of the 13th Iberoamerican congress on Pattern Recognition: Progress in Pattern Recognition, Image Analysis and Applications, pp. 22-29, 2008.

[9] M. I. Malik, M. Liwicki, L. Alewijnse, W. Ohyama, M. Blumenstein, B. Found, "ICDAR 2013 Competitions on Signature Verification and Writer Identification for On- and Offline Skilled Forgeries (SigWiComp 2013)", In Proc. of the ICDAR, pp. 1477-1483, 2013.

[10] J. Starck, E. J. Candès, D. L. Donoho, "The Curvelet Transform for Image Denoising," IEEE TIP, 11(6), pp. 670-684, 2002.

[11]S. Pal, U. Pal, M. Blumenstein, "A two-stage approach for English and Hindi off-line signature verification," In Proc. of the International workshop on Emerging Aspects in Handwritten Signature Processing, pp. 140-148, 2013.

[12] S. Pal, A. Alaei, U. Pal, M. Blumenstein, "Off-line Signature Verification based on Foreground and Background information," In Proc. of the International Conference on Digital Image Computing: Techniques and Applications, pp. 672-677, 2011.

[13] S. Pal, A. Alaei, U. Pal, M. Blumenstein, "Multi-Script Off-line Signature Identification," In Proc. of the International Conference on Hybrid Intelligent Systems, pp. 236-240, 2012.

[14] S. Pal, V. Nguyen, M. Blumenstein, U. Pal, "Off-line Bangla Signature Verification," In Proc. of the International Workshop on Document Analysis Systems, pp. 282-286, 2012.

[15]U. V. Marti, R. Messerli, H. Bunke, "Writer identification using text line based features," In Proc. of the ICDAR, pp. 101-105, 2001.

[16]L. Billard, E. Diday, "Symbolic Data Analysis: Definitions and Examples," Technical Report, 62 pages, 2003, at (http://www.stat.uga.edu/faculty/LYNNE/Lynne.html).

[17]H. N. Prakash, D. S. Guru, "Offline signature verification: an approach based on score level fusion", International Journal of Computer Applications, 1, pp.52-58, 2010.

[18]F. Alaei, N. Girard, S. Barrat, J. Y. Ramel, "A New One-Class Classification Method Based on Symbolic Representation: Application to Document Classification," In Proc. of the International Workshop on Document Analysis Systems, pp. 272-276, 2014.

[19]T. N. Vikram, K. Chidananada Gowda, R Urs Shalini, "Symbolic representation of Kannada characters for recognition," In Proc. of the International Conference on Networking, Sensing and Control, pp. 823826,2008

[20]C. Hertel, H. Bunke, "A Set of Novel Features for Writer Identification," pp. 679-687, 2003.

[21]S. H. Cha, S. Srihari. "Writer identification: statistical analysis and dichotomizer," Springer LNCS, 1876, pp. 123-132. 2000.

[22]S. H. Cha, S. Srihari. "Multiple feature integration for writer verification," In Proc. of the IWFHR, pp. 333-342. 2000. 